\title{
"Does it work?" A framework to evaluate the effectiveness of a robotic toy for children with special needs
}

\author{
E. Ferrari, B. Robins, and K. Dautenhahn
}

\begin{abstract}
To evaluate the performance of a social robot, both the aspects of safety and technical efficiency as well as the effectiveness of the interaction with the robot from the users' points of view need to be considered. The work described in this paper derived from the IROMEC ${ }^{1}$ project (Interactive Robotic Social Mediators as Companions) that investigates the design and role of an interactive, autonomous robotic toy in therapy and education contexts for children with special needs. The paper proposes a framework for the evaluation of robotic toys used as mediators for children with special needs and present its implementation with the specially designed IROMEC robot. Special attention is given to the interactions' effectiveness, considering the therapeutic and educational role that the robot can play for children with special needs in many different developmental areas.
\end{abstract}

\section{INTRODUCTION}

$\mathrm{O}^{\mathrm{r}} \mathrm{m}$ FTEN experiments conducted in the growing field of robotics focus mainly on the mechanical and technological aspects of the robots, their control algorithms or safety and compliance to standards which are usually carefully considered and analysed. When roboticists evaluate a robot then, in many cases, they are primarily interested in performance measures such as power consumption, accuracy and time to task completion [1]. Since robots are taking a growing part in human society, in many application areas that require human-robot interaction (e.g. entertainment, rehabilitation, therapy etc), experiments that intend to answer the question "Does it work?" do not only have to consider the technical, mechanical, and safety aspects, but need to extend the evaluation to the social role the robot might have and its long term effect on its users.

In recent years there have been many examples of robots being used in play activities of children with special needs, for therapeutic or educational purposes [2-11]. These robots have shown to be useful in promoting spontaneous play in children

E. Ferrari, B. Robins and K. Dautenhahn are with the Adaptive Systems Research Group. School of Computer Science, University of Hertfordshire, UK (email: \{e.ferrari, b.robins, k.dautenhahn\}@herts.ac.uk)

\footnotetext{
${ }^{1}$ The work described in this paper was primarily conducted within the EU project IROMEC (Interactive Robotic Social Mediators as Companions) and was funded by the European Commission in the 6th Framework Program under contract IST-FP6-045356. Partial support was provided by the European Commission under contract number FP7-231500-RoboSkin
}

with developmental disorders, engaging them in playful interactions. Many different research methods were used to conduct evaluations, pointing out the need for a shared framework that would help the process of execution and integration of research results.

This paper presents a framework to evaluate the effectiveness of a robotic toy targeting children who are prevented from or inhibited in playing. The framework has been developed and applied within the IROMEC project.

\section{THE FRAMEWORK - STAGES IN ROBOT EVALUATION}

The proposed framework for an effective evaluation of such social robots includes three stages: the technical evaluation of the robot, the usability evaluation and the evaluation of the possible effects that the interaction with the robot might have on the users.

\section{A. Technical evaluation}

First, tests to determine the technical status of the robot need to be carried out in a certified laboratory. This evaluation consists of various tests to examine the robot's mechanical, plastic and electronic components to ensure its adequacy, safety and compliance to international standards. Hazard and Operability analysis (HAZOP) can be used whereby mechanical and physical properties are analyzed in helping to identify both direct hazards as well as operability problems that potentially can lead to process hazards.

\section{B. Usability study}

The term usability refers to the ease of use of an object, or more precisely "the extent to which a product can be used by specified users to achieve specified goals with effectiveness, efficiency and satisfaction in a specified context of use" [12]. Usability evaluation has the great value in highlighting the elements that need to be changed in order to improve the quality of the interaction with the robot.

To enable a robot to acquire the role of an interactive robotic social mediator for children with special needs, it needs to be accepted by the children as an integral element of the play activity. In this context, usability evaluation of the robot needs to be conducted first with secondary users (adults and typically developing peers) and subsequently with children from the target user groups (children with special needs). The 
evaluation should focus on general usability aspects of the interaction with the robot such as position, colors and size of all robot's elements (buttons, icons, head, eyes, etc), overall appearance of the robot, sound features (audio feedback and general sound of the robot's motors), battery issues (position, weight and time to recharge), quality of the robot's visual feedback and movements, the screens' visibility and GUI (graphical user interface) analysis. Also, this phase should be used to assess the play scenarios, considering any difficulties that may arise during their performance.

\section{Effectiveness of the interaction}

This step of the framework is the one that requires more effort due to the direct and/or indirect involvement of the users. In order to evaluate the effect that the interaction with a robotic toy might have on children with special needs and its potential in improving their abilities in different developmental areas, an assessment of the children's characteristics in those areas before and after the intervention is needed. This assessment necessitates to be comprehensive identifying the broad range of the child's strengths and needs and covering all important interaction domains (if important domains are not included then the robot's effectiveness may be reduced). Assessment that involves children with special needs necessitates to be more flexible than the typical school-aged assessment method in which the child is asked to answer questions or to perform tasks [13]. In particular the evaluation of the effectiveness of the intervention must overcome the communication impairments generally exhibited by many children with special needs.

There is currently a vast array of standardized evaluation tools that may help to assess children from various user groups. Therefore, a comprehensive review of the literature is required in order to determine the most appropriate evaluation tool that can be used to evaluate the progress of the children. The main characteristic of an effective therapeutic and educational evaluation is that it is comprehensive, covering all the important areas of children's development, seeing the child as a whole and identifying the broad range of the child's strengths and needs. If a tool to assess the children and to evaluate the effects of the interaction with the robot is not available or its application is judged as not satisfactory (e.g. not covering the whole range of specific objectives relevant to the particular user group), then a new specific tool should be designed and a triangulation technique can be applied, i.e. the use of more than one method for gathering data in order to enhance confidence in the findings. This is certainly not a new approach in social sciences research [14], and it has been discussed extensively in the psychology field [15]. Increasingly, more evaluations are relying on mixed methods, recognizing that both quantitative and qualitative approaches are valuable and have something unique to offer. The quantitative method can show, using pre and post tests, what changes have occurred and how generally and frequently they occurred; while the qualitative method can reveal in detail how changes occurred in day-to-day activities. By combining the qualitative and quantitative methods, the weakness of each single method can be overcome and a more complete picture of a child's achievements and possible progresses can be obtained.

Questionnaires and surveys are examples of quantitative research instruments consisting of a series of questions (items) which have the purpose of gathering statistically analyzable data. Many traditional questionnaires that target children use a format that requires the child to respond to standardized stimuli in a formal testing session. Using this approach to measure a child's ability and behaviour may be inaccurate (e.g. a child might choose not to respond to stimuli because of unfamiliarity with the testing situation, inability to understand what is required, discomfort, etc), especially when the users are children with special needs. In contrast, choosing as a respondent the adult (a teacher or a therapist) who knows the child well, does not require the child to perform a specific task at a specific time and it has the advantage of producing valid measurements of the day-to-day activities that are hard to be adequately measured through direct administration of tasks.

Observations and interviews are qualitative research techniques, which allow a detailed investigation of a specific topic. Their aim is to get increased insight on respondents' knowledge on important issues.

The next section gives a short description of the project in which the framework has been developed and applied in.

\section{IROMEC PROJECT}

The IROMEC project [16] recognizes the important role of play in child development and targets children with special needs providing them with opportunities for learning and improving in different developmental areas, while they have fun. A key outcome of the project was a novel robot prototype. The robotic system can engage children with special needs in play activities, involving other children as well as adults. The IROMEC robot is not meant to replace teachers or therapists; it is a tool for social mediation. The robotic toy is intended to be used as a scaffolding element during child development by encouraging children to discover a range of play styles, from solitary to social and cooperative play.

The target user groups of the project are children with special needs, and in particular children with:

- autism (AUT): autistic spectrum disorder is a developmental disorder characterized by impairments in communication, social interaction, and imagination that can occur to different degrees and in a variety of forms 
[17-18]. Children at the lower end of the spectrum have been considered part of the AUT group;

- mild mental retardation (MMR), also referred to as intellectual disabilities or learning disabilities. Children from this group experience difficulties in the dimensions of intellectual abilities, adaptive behaviour, participation, interactions and social roles, health and context [19]. Children with an IQ ranging from 50-55 to approximately 70 [18] have been considered part of the MMR group;

- severe motor impairment (SMI) concerns children who are limited in their ability to play due to limitations in their movement, if they are able to move at all. Independent of the cause of the motor impairment, children who can access technology only by using a scanning technique [20], have been considered part of the SMI group.

Ten IROMEC play scenarios for robot assisted play and robotic mediator have been developed, adopting the ESAR system [21] that identifies four different types of play (fig.1).

Play scenarios have been developed taking children's specific strengths and needs into consideration and covering five developmental areas ${ }^{2}$ : sensory development, cognitive development, communicational and interaction, motor development, and social and emotional development. A comprehensive set of therapeutic and educational objectives has been identified working closely with therapists and teachers (see appendix). These objectives have been developed according to the ICF-CY classification (International Classification of Functioning, Disability and Health for Children and Youth) [22]. For a more detailed description of the robot play scenarios see [23, 24].

Knowing that the children from the different target user groups have different requirements, the IROMEC robotic toy has been developed as a modular system that can be tailored to the users' needs. A specific feature of the robot is that it can be adapted to various play scenarios by being modular in terms of software (for activation of different behaviours) and hardware (by attaching different interaction modules).

The IROMEC robot (see fig. 2) has two different possible configurations: horizontal and vertical [26]. In the horizontal configuration the interaction module is attached to the mobile platform in order to support a set of activities requiring a wider mobility of the robot. In the vertical configuration the interaction module is connected to a dedicated docking station to provide both stability and recharging. Additionally, the robot has several interfaces such as dynamic screens for input and output, buttons and wireless switches. The IROMEC robot operates autonomously once a particular play scenario has

\footnotetext{
${ }^{2}$ Detailed information on the IROMEC system and the five developmental areas can be found in [25].
}

been selected and it can adjust over time by gradually increasing
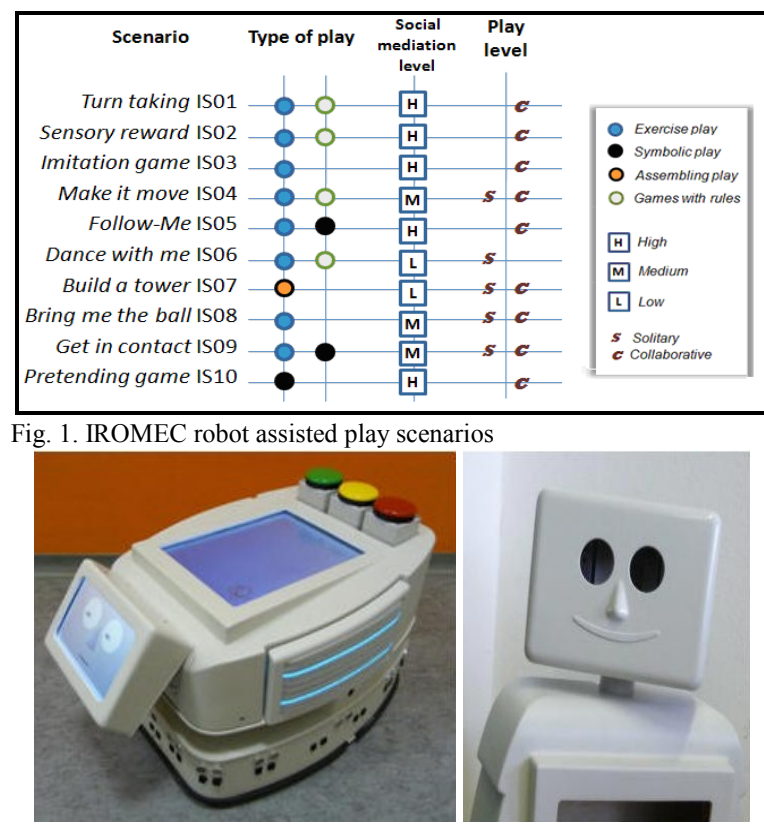

Fig. 2. The IROMEC robot in its horizontal (without optional mask) and vertical (with optional mask) configurations

the complexity of the scenario played. Thus, small behaviour variations could help children to improve their abilities in different developmental areas. For a more detailed description of the robot's technical characteristics, see [26, 27].

The next section gives a short description of the application of the framework to evaluate the effectiveness of the interaction with the IROMEC robot.

\section{FRAMEWORK IMPLEMENTAION: THE CASE OF THE IROMEC ROBOT}

During the IROMEC project, among other technical tests the EN 62115:2006 test for "Electric toys - Safety" and the EN 71:2005 test for "Safety of Toys" and "Mechanical and Physical Properties" (except those tests that could deteriorate the sample) have been conducted.

In addition to a usability evaluation, it has been considered important to investigate the playfulness that children experienced during the interaction with the IROMEC robot. The assessment has been conducted using the widely accepted Test of Playfulness [24]. A positive effect regarding the level of playfulness is an indication of the suitability of the robot and its acceptance by the children in their play activities, as well as of its potential in achieving positive therapeutic and educational effects.

In order to evaluate the effect that interaction with the 
IROMEC robot might have on the children, first an appropriate tool needed to be identified. A literature analysis about existing standardized, validated and used tools for the evaluation of children's improvements in the five developmental areas has been conducted. It emerged that there are a variety of assessment instruments available - from cognitive development to overall functioning - most of which are not primarily designed for children whose development is markedly delayed, as is the case for the IROMEC target user groups. In addition these tools are often highly detailed, focusing on one single and specific aspect of child development and consider only one or two of the five developmental areas, while the other areas remain un-assessed [28-39].

It was concluded that none of these tests alone are directly applicable to assess all of the possible improvements targeted by the objectives of the play scenarios of the IROMEC robot. Two or more tools were needed to cover all five different developmental areas. Considering the amount of time required to complete each of the available tools (on average 40 minutes for each child), the solution to apply two or more of these tools for the evaluation of a child's improvement was considered neither practical in a busy school environment nor satisfactory. Also, almost all of these tools have been standardized for typically developing children, and the applicability of the test is determined by the chronological age of the child rather than by cognitive or developmental ability (e.g. the Bayley Scales of Infant Development covers the age range 1 to 42 months; the WPPSI covers the age range 3 years to 7 years 3 months). Besides, the assessment of children from our target user group can also present problems that are not ordinarily associated with assessing typically developing children, for example, poor linguistic skills and attention problems. For all these reasons these tools have been considered not suitable to be applied to the evaluation of the effects that the interaction with the IROMEC robot might have on the children.

Therefore, there was the need to design a specific evaluation tool, appropriate for each user group, covering all the identified therapeutic and educational objectives in all the developmental areas.

\section{A. IROMEC evaluation tool}

The first stage in designing the IROMEC effectiveness evaluation tool was a consultation with education and rehabilitation experts in order to discuss a) the various therapeutic and educational objectives of child development that can be related to playing with the IROMEC robot and b) the instruments for the evaluation of children's improvements in areas of sensory development, communicational and interaction, motor development, cognitive development and social and emotional development (see appendix).

According to the triangulation technique suggested by the framework, quantitative (questionnaire) and qualitative (observation grid and a semi-structured interview) instruments have been developed to evaluate the effect that interaction with the IROMEC robot might have on the children.

\section{1) Questionnaire}

The IROMEC questionnaire covers all the five different developmental areas. Its design process as a tool for evaluating improvements of children who interacted with the IROMEC robot went through several stages. First of all, we considered more effective to focus on the child's performance compared to the child's ability. While ability is a quality that allows a person to achieve or to accomplish a task (e.g. a child is able to move his arms to imitate someone else's arm movements), performance demonstrates the actual ability when required (e.g. the child imitates arm movements). In order to include the correct items in a questionnaire, it is necessary to define what exactly is being investigated. First, each objective from any of the five developmental areas was given an operational definition (e.g. an explicit statement that defines the objective). The questionnaire items were formulated around these definitions and with reference to available tools (literature analysis and experts' advice). Items in the questionnaire were written in short simple and direct language thus making the questionnaire clear and easy to use also by non-clinical users (e.g. teachers, parents).

The items were discussed with experts in a reviewing process and edited accordingly, and the final questionnaire was subjected to a pre-test phase. This was followed by a revision process using a "questionnaire revision form" that was specially developed for this purpose and was completed by the various project partners. This process helped in gathering information to improve the questionnaire. The final version of the IROMEC evaluation questionnaire consists of a list of questions (items) that are presented to the adult who is familiar with the child (see fig. 3). They are asked to answer the questionnaire twice during the evaluation process: at the beginning (as a baseline) and at the end of the therapeutic or educational intervention. The number of items varies according to the child's target group, the scenarios s/he played, and the related objectives. Therefore the time required to complete the questionnaire varies accordingly, from a minimum of 30 to a maximum of 60 minutes. The respondent has to rate each item of the questionnaire according to five categories: never, rarely, occasionally, frequently and always. The response categories represent a progression between the lowest level of response and a higher one and provide a method of obtaining valid information about an individual's on-going behavior. Next to each question, some space is left available for the respondents to make comments. Leaving space for comments provides valuable information not captured by the response categories and makes the questionnaire easier to complete. 


\begin{tabular}{|c|c|}
\hline \multicolumn{2}{|r|}{ COGNITIVE DEVELOPMENT } \\
\hline Sub-area & ying \\
\hline Objective & MIRROR AND IMITATE SIMPLE AND COMPLEX MOVEMENTS \\
\hline \multicolumn{2}{|c|}{$\begin{array}{l}\text { The child... } \\
\text {... engages in imitation spontaneously }\end{array}$} \\
\hline \multicolumn{2}{|c|}{... engages in imitation when prompted } \\
\hline \multicolumn{2}{|c|}{$\begin{array}{l}\text {... imitates simple gesture (gesture that s/he can see self perform, such as } \\
\text { clapping, waving) }\end{array}$} \\
\hline \multicolumn{2}{|c|}{... responds to imitation of his/her actions with further repetition } \\
\hline \multicolumn{2}{|c|}{... initiates and pauses to allow imitation in social play } \\
\hline \multicolumn{2}{|c|}{... imitates more complex gestures on cue } \\
\hline \multicolumn{2}{|c|}{$\begin{array}{l}\text {... demonstrates a delay response, but } \mathrm{s} / \mathrm{he} \text { is able to replicate another } \\
\text { person's actions (timing of response in imitations) }\end{array}$} \\
\hline
\end{tabular}

Fig. 3. List of items from the IROMEC evaluation questionnaire related to the objective "Mirror and imitate simple and complex movements", targeting children of the AUT group

\section{2) Observation grid and semi-structured interview}

To support the information obtained through the use of the questionnaire, an IROMEC observation grid has been developed to be used during each session to collect any important information or improvement related to the child's behaviour in the developmental areas. While the questionnaire was filled in twice (before starting the intervention and at the end of the intervention period), the observation grid was completed after each play session. In that way the teacher, therapist or educator had a tool that assists in keeping records of all important events that happened during a play session, helping to identify any play patterns (e.g. a behaviour that occurs during specific activities) and to investigate any factors that are reinforcing that behaviour and which might support changes or improvements in the child's skills.

The IROMEC semi-structured interviews have been conducted at the end of the intervention, after each child played with the IROMEC robot over an extended period of time (8-10 sessions on average, over 2 months). The respondent was the adult who knew the child and participated in the interaction play activity. The list of topics that the IROMEC researcher has discussed during the interview was related to the developmental areas and in particular to those areas where the results from the observation grids and questionnaire showed changes in child behavior. In addition, some general aspects of the overall experience of the children and their carer during the interaction with the robot also were discussed.

\section{3) IROMEC Software}

A software was developed to further facilitate the adult user during the intervention period and help the evaluation process. This software assists in setting the objectives for each child, planning the intervention process, choosing the relevant play scenarios to be played in each session (according to the selected objectives), monitoring the child's progress and assist the evaluation. The advantage of using the software is that it no longer requires to complete a hard copy of the questionnaire, containing all the questions. Instead the adult selects from a list the objectives relevant to the specific child and the play scenarios on which the intervention focuses. Next, a list of selected questions related to the chosen objectives is presented to the respondent (evaluation baseline). For each question a column dedicated to comments is provided. The respondent can use this column to add any additional information (qualitative data) that might be useful. For example, the respondent may wish to note down the context in which the child demonstrates a skill when achieving it only occasionally. At the end of the intervention the post evaluation questionnaire, containing the same questions as in the base-line evaluation, is completed and a comparison of the results from the two questionnaires (pre/post evaluation) is reported highlighting any changes in scores.

\section{DISCUSSION}

As robots are expected to become more and more part of our society, the evaluation of the effects of the interaction with them becomes increasingly important. The evaluation of robots' technical, mechanical and safety aspects are as much important as the evaluation of their effects on the users that interact with them. Studies have showed that robots can have positive effects on children with special needs, but there is still a need for a common methodology on how to evaluate this aspect. The work presented in this paper can benefit researchers as it presents a general framework to analyze the effectiveness of a play robotic system for children with special needs, focusing in particular on the evaluation of the effects of the interaction. In addition, the work presents an example of the framework applied to the evaluation in the IROMEC project. The paper describes the evaluation process of the IROMEC robotic toy. Although the stage related to the evaluation of the effects of the robot, carried on in different European countries (UK, Italy and Austria), has not been completed yet, first data collected suggest that the IROMEC robot has the potential to be a valuable tool for children with special needs helping them to experience different and new play interactions and encouraging the development of skills in various developmental areas. This work could be useful in other applications involving human-robot interaction, especially in applications that targets people with special needs using robots for therapeutic or educational purposes.

\section{BEYOND IROMEC}

The framework outlined in this paper will be used in the RoboSkin (Skin-Based Technologies and Capabilities for Safe, Autonomous and Interactive Robots) European project which has started in 2009. The project aims at developing and demonstrating a range of new robot capabilities based on the tactile feedback provided by novel robotic skin technology. 
One project objective concerns the use tactile feedback to improve human-robot interaction capabilities in the application domain of robot assisted play for children with autism. Here, the framework described in this paper will be highly valuable in order to assess the effectiveness of a robot equipped with skin technology, using the minimally expressive humanoid robot KASPAR [40].

\section{APPENDIX}

The IROMEC therapeutic and educational objectives are objectives for child development and have been developed in consultation with the panels of experts and according to the ICF-CY classification [22]. The IROMEC robot offers the children different play scenarios which provide a variety of experiences and possibilities for developing aspects in all areas of child development. However it is important to note that children do not develop their skills in isolation from each other, and that the abilities they gain might overlap in different areas (e.g. cognitive, social and emotional development). In the IROMEC project we have classified the objectives into five areas of child development: sensory development, communication and interaction, cognitive development, motor development, and social and emotional development. This division is somewhat artificial but is necessary in order to help the teacher or therapist to focus on the intervention. It allows them to choose out of the long list of objectives (see table below) the one(s) to consider in their intervention.

IROMEC developmental areas, sub-areas and objectives.

\begin{tabular}{|c|c|c|}
\hline $\begin{array}{l}\text { Dev. } \\
\text { area }\end{array}$ & Sub-area & Objectives \\
\hline \multirow{4}{*}{ 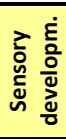 } & \multirow{4}{*}{ Perceptual functions } & Visual perception \\
\hline & & Tactile perception \\
\hline & & Visuospatial percep. (spatial awareness) \\
\hline & & Proprioception (body awareness) \\
\hline \multirow{18}{*}{ 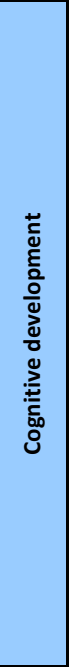 } & \multirow{2}{*}{$\begin{array}{l}\text { Energy and drive } \\
\text { functions }\end{array}$} & Improve motivation to act \\
\hline & & Feel in control \\
\hline & Global intellect. fun. & Understand cause \& effect \\
\hline & Memory functions & Short term memory \\
\hline & \multirow{4}{*}{$\begin{array}{l}\text { Higher-level } \\
\text { cognitive functions }\end{array}$} & Abstraction \\
\hline & & Organization and planning \\
\hline & & Cognitive flexibility \\
\hline & & Control of the wish for or delay of gratification \\
\hline & Copying & $\begin{array}{l}\text { Mirror and imitate simple and complex } \\
\text { movements }\end{array}$ \\
\hline & \multirow{3}{*}{$\begin{array}{l}\text { Learning through } \\
\text { action with objects }\end{array}$} & Ability to carry on actions relating objects \\
\hline & & Ability to carry on actions involving pretence \\
\hline & & $\begin{array}{l}\text { Ability to engage in make-believe activities } \\
\text { involving imaginary person }\end{array}$ \\
\hline & \multirow{6}{*}{ Attention } & Focusing attention \\
\hline & & Attend to the human touch, face $\&$ voice \\
\hline & & Changes in the environment \\
\hline & & Maintain attention \\
\hline & & Shifting attention \\
\hline & & Dividing attention \\
\hline
\end{tabular}

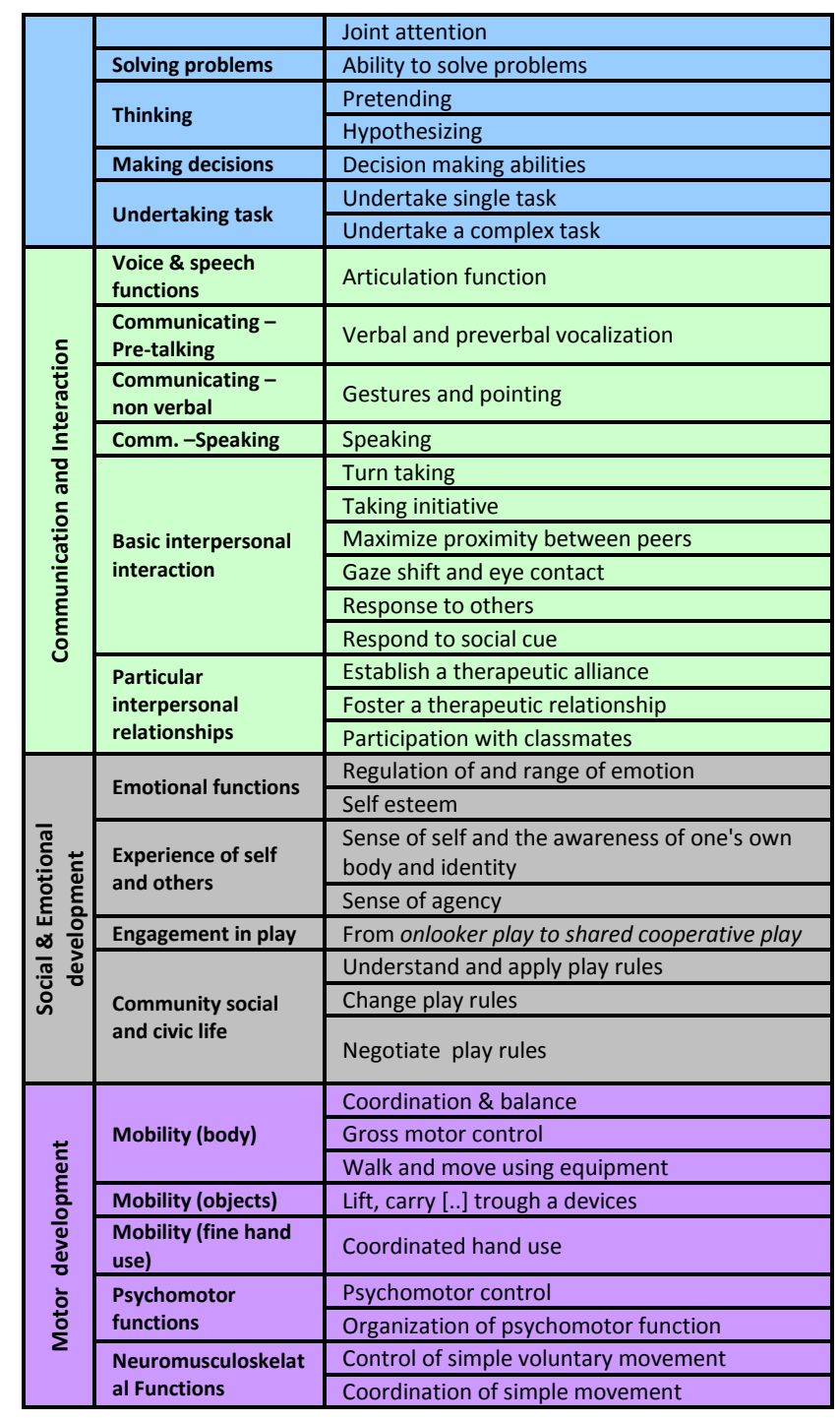

\section{REFERENCES}

[1] K.M. Tsui, and Yanco H.A., "Towards establishing clinical credibility for rehabilitation and assistive robots through experimental design", Workshop on Good experimental methodology in robotics, Robotics Science and Systems, 2009, Seattle, WA, USA

[2] H. Kozima et al, "Children-robot interaction: A pilot study in autism therapy", in Prog. Brain Res, 2007, 164, pp. 385-400.

[3] C.E. Lathan, and S. Malley, "Development of a New Robotic Interface for Telerehabilitation", in Proc. 2001 EC/NSF workshop on Universal accessibility of ubiquitous computing. 2001, pp. 80-83.

[4] P. Marti, A. Rullo, H. H. Lund, and J. Nielsen, "Playing with emotions" , in Proceedings of XII European Conference on Cognitive Ergonomics, D.J. Reed, G. Baxter, M. Blythe (eds.) York, UK, 2004.

[5] P. Marti, A. Pollini, A. Rullo, and T. Shibata, "Engaging with artificial pets", in EACE 2005.

[6] D. Stiehl, J. Lieberman, C. Breazeal, L. Basel, R. Cooper, and H. Knight, "The Huggable: A Therapeutic Robotic Companion for Relational, Affective Touch", in IEEE Consumer Communications and Networking Conf., Las Vegas, NV, USA, 2006. 
[7] B. Robins, K. Dautenhahn, R. te Boekhorst, and A. Billard, "Effects of repeated exposure of a humanoid robot on children with autism," in Designing a More Inclusive World, S. Keates, J. Clarkson, P. Langdon, and P. Robinson, Eds. London: Springer- Verlag, 2004, pp. 225-236.

[8] B. Robins, P. Dickerson, P. Stribling, and K. Dautenhahn, "Robotmediated joint attention in children with autism: A case study in a robot human interaction," Interaction studies, 2004, 5, pp. 161-198.

[9] B. Robins, K. Dautenhahn, R. te Boekhorst, and A. Billard, "Robotic assistants in therapy and education of children with autism: Can a small humanoid robot help encourage social interaction skills?, ” in Universal Access in the Information Society, Springer-Verlag, 2005, pp. 105-120.

[10] F. Michaud, H. Larouche, F. Larose, T. Salter, A. Duquette, H. Mercier, M. Lauria, "Mobile robots engaging children in learning", in Canadian Medical and Biological Engineering Conf., Toronto, 2007.

[11] B. Robins, K. Dautenhahn, P. Dickerson, "From Isolation to Communication: A Case Study Evaluation of Robot Assisted Play for Children with Autism with a Minimally Expressive Humanoid Robot ", Proc. Second Inter. Conf. Advances in CHI, ACHI09, 2009, 205-211.

[12] ISO 9241-11, Ergonomic requirements for office work with visual display terminals - Part 11: Guidance on usability, International Organization for Standardization, 1998.

[13] M.M. Schmidt, "Using Play Therapy Assessment in an Elementary and Intermediate School Setting”, in School-Based Play Assessment pp. 315.

[14] T.D. Jick, "Mixing Qualitative and Quantitative Methods: Triangulation in Action", Administrative Science Quarterly, 1979, 24, 602-611.

[15] V.J. Caracelli and J.C. Greene, "Crafting Mixed-Method Evaluation Design" In J. C. Greene and V. Caracelli (eds.), Advances in MixedMethod Evaluation: The Challenges and Benefits of Integrating Diverse Paradigms. New Directions for Program Evaluation, 1997, San Francisco: Jossey-Bass

[16] www.iromec.org

[17] R. Jordan (1999). Autistic Spectrum Disorders - An Introductory Handbook for Practitioners. London: David Fulton Publishers.

[18] DSM-IV-R (2000). Diagnostic and Statistical Manual of Mental Disorders. American Psychiatric Association

[19] R. Luckasson, S. Borthwick-Duffy, W.H.E. Buntinx, D.L. Coulter, E.M. Craig, A. Reeve, R.L. Schalock, M.E. Snell, D.M. Spitalnick, S. Spreat, M.J. Tasse, "Mental retardation: Definition, classification, and systems of supports 10th Edition". American Association on Mental Retardation Washington DC, 2002.

[20] S. Besio, "Allowing Children with Motor Impairment to Play, Learn, Learn to Play: A new Biennial Research Project of SIVA", in Proc. 3rd AAATE Conf., Ljubljana, 2001, pp. 231-234.

[21] D. Garon, R. Filion, and M. Doucet, El sistema ESAR: Un método de análisis psicológico de los juguetes. Alicante, Spain, 1996.

[22] WHO, International Classification of Functioning, Disability and Health. Geneva: World Health Organization, 2001.

[23] B. Robins, E. Ferrari, and K. Dautenhahn, "Developing Scenarios for Robot Assisted Play", in Proc. 17th International Symposium on Robot and Human Interactive Communication RO-MAN2008, 2008, pp. 180186.

[24] A. Bundy, "Play and playfulness", in: D. Parham \& L. Fazio (Eds) Play in occupational therapy for children, St Louis, MO, Mosby-Year Books, 1997.

[25] E. Ferrari, B. Robins and K. Dautenhahn, "Therapeutic and educational objectives in Robot Assisted Play for children with autism”, in Proc. 18th International Symposium on Robot and Human Interactive Communication RO-MAN2009, 2009, pp.108-114.

[26] B. Robins, E. Ferrari, K. Dautenhahn, G. Kronrief, B. Prazak-Aram, G. Gerderblom, F. Caprino, E. Laudanna, "Developing Scenarios for Robot Assisted Play Informed by User Panels and Field Trials”, in International Journal of Human-Computer Studies IJHCS (in press).

[27] P. Marti, C. Moderini, L. Giusti, A. Pollini, "A Robotic Toy for Children with special needs: From requirements to Design" in Proc. IEEE 11th
International Conference on Rehabilitation Robotics ICORR, 2009, pp. 918-923.

[28] N. Bayley, "The Bayley Scales of Infant Development", second edition, The Psychological Corporation, San Antonio, Texas, 1993.

[29] D. Wechsler, "Wechsler Preschool and Primary Scale of IntelligenceRevised", San Antonio, TX: Psychological Corporation, 1989.

[30] D. Wechsler, "Wechsler Intelligence Scale for Children-Third Edition", San Antonio, TX: Psychological Corporation, 1991.

[31] C. Westby, "A scale for assessing children's pretend play", in: C. E. Shaefer, K, Gitlin \& A. Sandgrund (Eds) Play diagnosis and assessment (New York, John Wiley \& Sons), 131-161, 1991.

[32] Ulrich, D.A. "Test of Gross Motor Development.", Austin, TX: PROED, 1985.

[33] S. S. Sparrow, A.S. Carter, and D.V. Cicchetti, "Vineland Screener: Overview, Reliability, Validity, Administration, and Scoring.", New Haven, CT: Yale University Child Study Center, 1993.

[34] T.M. Stephens and K.D. Arnold "Social Behavior Assessment Inventory: Professional manual”. Odessa, FL: Psychological Assessment Resources, 1992.

[35] E.M. Mullen "Mullen Scales of Early Learning", (AGS ed.). Circle Pines, MN: American Guidance Service Inc, 1995.

[36] C. Lord, M.M. Rutter and A. Le Couteur "Autism Diagnostic InterviewRevised: A Revised Version of a Diagnostic Interview for Caregivers of Individuals with Possible Pervasive Developmental Syndromes", Journal of Autism and Developmental Syndromes 24 pp. 659-85, 1994.

[37] T.W Linder, "Transdisciplinary Play-Based Assessment: A Functional Approach to Working with Young Children"; Revised Edition, Paul H. Brooks Publishing Co., Inc, 1993.

[38] S.I. Greenspan, G. Degangi and S. Wieder, "Functional Emotional Assessment Scale: Clinical and Research Applications". Bethesda, MD: Interdisciplinary Council on Developmental and Learning Disorders, 2001.

[39] R.R. Fewell and J.S Rich, "Play assessment as a procedure for examining cognitive, communication, and social skills in multihandicapped children". Journal of Psychoeducational Assessment, 2, pp. 107-118, 1987.

[40] K. Dautenhahn, C.L. Nehaniv, M.L. Walters, B. Robins, H. Kose-Bagci, N. Assif Mirza, and M. Blow, "KASPAR - A Minimally Expressive Humanoid Robot for Human-Robot Interaction Research”, Special Issue on "Humanoid Robots", Applied Bionics and Biomechanics, vol. 6, 3, pp. 369-397, 2009. 